unbound fraction, which is responsible for the antiepileptic effect, may be increased, ${ }^{19}$ and serum protein binding is decreased later in pregnancy. ${ }^{20}$ Unfortunately, direct measurement of the free concentration of antiepileptic drugs is not yet generally available or reliable enough for routine use. The drug dosage should therefore be increased cautiously if major fits recur during pregnancy, and the patient's condition reviewed with the blood picture checked and serum concentrations of drugs measured monthly-or more frequently if fits are not controlled. ${ }^{14}$

A woman having treatment for epilepsy who wants to embark on pregnancy should ideally be on a single drug with its serum concentration in the optimum range. Nevertheless, if control has been achieved with a combination of antiepileptic drugs the patient should be encouraged to continue with the same regimen, because the risks to the fetus and mother may be greater from uncontrolled fits-and particularly from status epilepticus-than from the drugs. Weight gain should be restricted, fluid retention prevented, and iron and low-dose folic acid supplements should be given to prevent or treat anaemia. Toxic effects of the antiepileptic drugs, including the haematological complications, should be prevented or treated promptly in both the mother and the newborn baby. Vitamin $\mathrm{K}$ should be given and expert neonatal care should be available. Careful follow-up post partum with serial measurements of serum drug concentrations is necessary, as it may take six to eight weeks for the prepregnancy state to be restored. Close collaboration with the obstetrician is important and further studies of the effects of pregnancy on epilepsy are required.

Fits occurring only in pregnancy or the puerperium with no evidence of toxaemia have been described as gestational epilepsy. ${ }^{21} 22$ This is a term, however, that should be applied only in retrospect: no one can predict that fits will not recur unrelated to pregnancy, or that they will recur in subsequent pregnancies. In one series ${ }^{23} 159$ out of 441 women developed epilepsy between the ages of 16 and 40; inevitably some women will have their first-ever fit during pregnancy. If it occurs without either toxaemia or other cause antiepileptic treatment can be withheld unless there is a second fit. Investigations should include examination of the blood picture to see if there is anaemia, and electroencephalography. A normal electroencephalogram or one with non-specific abnormalities only is of no positive or negative help with the diagnosis, but generalised paroxysmal discharges on the electroencephalogram suggest idiopathic epilepsy. If there are clinical or electroencephalographic features suggesting a focal or structural cause-which is not uncommon in women who develop epilepsy in pregnancy-further investigations will probably be required if fits recur or if the neurological signs persist. Nevertheless, as radiography and computed tomography are done in pregnancy only if absolutely necessary, the timing will depend on several factors, including the stage of pregnancy and the likely cause. Driving must be stopped after a fit, in accordance with the advice given in Medical Aspects of Fitness to Drive. ${ }^{24}$

${ }^{1}$ Johannessen SI, Morselli PL, Pippenger CE, Richens A, Schmidt D, Meinardi $\mathrm{H}$, eds. Antiepileptic therapy: advances in drug monitoring. New York: Raven Press, 1980.

2 Shorvon S, Reynolds EH. Reduction in polypharmacy for epilepsy. Br Medf 1979;ii:1023-5.

${ }^{3}$ Coulam CB, Annegers JF. Do anticonvulsants reduce the efficacy of oral contraceptives? Epilepsia 1979;20:519-25.

4 John A. Epilepsy and the pill. Br Med F 1976;ii:528.

5 Hopkins A. Epilepsy in adults. Medicine 1980;32:1632-40

${ }^{6}$ Zackai EH, Mellman WJ, Neiderer B, Hanson JW. The fetal trimethadione syndrome. $\mathcal{F}$ Pediatr $1975 ; 87: 280-4$.
7 Feldman GL, Weaver DD, Lovrien EW. The fetal trimethadione syndrome. Report of an additional family and further delineation of this syndrome. Am $\mathcal{F}$ Dis Child 1977;131:1389-92.

8 Annegers JF, Elveback LR, Hauser WA, Kurland LT. Do anticonvulsants have a teratogenic effect ? Arch Neurol $1974 ; 31: 364-73$.

9 Janz D. The teratogenic risk of antiepileptic drugs. Epilepsia 1975;16: 159-69.

${ }^{10}$ Nakane Y. Congenital malformation among infants of epileptic mothers treated during pregnancy-the report of a collaborative study group in Japan. Folia Psychiatr Neurol fpn 1979;33:363-9.

11 Stumpf DA, Frost M. Seizures, anticonvulsants, and pregnancy. Am $\mathcal{F} D$ is Child 1978;132:746-8.

12 Weber M, Schweitzer M, Mur J-M, Andre M, Tridon P, Vert P. Epilepsie, médicaments antiépileptiques, et grossesse. Arch Fr Pediatr 1977;34: 374-83.

13 Donaldson JO. Neurology of pregnancy. Philadelphia: WB Saunders, 1978.

${ }^{14}$ Montouris GD, Fenichel GM, McLain W. The pregnant epileptic: a review and recommendations. Arch Neurol 1979;36:601-3.

15 Knight AH, Rhind EG. Epilepsy and pregnancy: a study of 153 pregnancies in 59 patients. Epilepsia 1975;16:99-110.

${ }^{16}$ Lander CM, Edwards VE, Eadie MJ, Tyrer JH. Plasma anticonvulsant concentrations during pregnancy. Neurology (Minneap) 1977;27:128-31.

${ }^{17}$ Landon MJ, Kirkley M. Metabolism of diphenylhydantoin (phenytoin) during pregnancy. Br f Obstet Gynaecol 1979;86:125-32.

18 Bossi L, Assael BM, Avanzini G, et al. Plasma levels and clinical effects of antiepileptic drugs in pregnant epileptic patients and their new-borns. In: Johannessen SI, Morselli PL, Pippenger CE, et al, eds. Antiepileptic therapy: advances in drug monitoring. New York: Raven Press, 1980: 9-14.

${ }^{19}$ Dam M, Christiansen J, Munck O, Mygind KI. Antiepileptic drugs: metabolism in pregnancy. Clin Pharmacokinet 1979;4:53-62.

${ }^{20}$ Ruprah M, Perucca E, Richens A. Decreased serum protein binding of phenytoin in late pregnancy. Lancet 1980 ;ii:316-7.

${ }^{21}$ Burnett CWF. A survey of the relation between epilepsy and pregnancy. Fournal of Obstetrics and Gynaecology of the British Empire 1946;53: 539-56.

${ }^{22}$ Dimsdale H. The epileptic in relation to pregnancy. $\mathrm{Br} \mathrm{Med} \mathcal{F} 1959$;ii: 1147-50.

${ }^{23}$ Turner WA. Epilepsy; a study of the idiopathic disease. London: Macmillan, 1907.

${ }^{24}$ Raffle A, ed. Medical aspects of fitness to drive. 3rd ed. London: Medical Commission on Accident Prevention, 1976.

\section{Steroids in bronchitis}

Corticosteroids have dramatically improved the treatment for bronchial asthma, ${ }^{1}$ but their value in chronic bronchitis remains a matter of dispute. While some trials have shown a favourable response, ${ }^{2}$ others have failed to show any improvement. $^{3}$ Interpreting these findings is difficult, since most of the trials were not double blind and did not include controls. 4

Albert and his colleagues 5 in Seattle, however, have recently reported a well-designed trial to investigate the effect of methylprednisolone in chronic obstructive airways disease. Patients were admitted to the study if they had evidence of chronic airflow obstruction (a forced expiratory volume in one second of $60 \%$ or less than the predicted value of $60 \%$ or less than a simultaneous forced vital capacity). These measurements were taken after administration of a bronchodilator and at a time of clinical stability. Objective assessments made before and after treatment consisted of bedside spirometry (again after a bronchodilator) and measurement of the blood gases in arterial blood. Patients with a history of asthma or reversible bronchospasm were excluded. Methylprednisolone was given intravenously at a dose of $0.5 \mathrm{mg} / \mathrm{kg}$ every six hours (or an identical placebo) and added to a standard regimen of intravenous aminophylline, nebulised isoproterenol, antibiotics, and oxygen, thus avoiding the possibility that differences between the two groups could be attributed to omission of routine forms of treatment. Patients were allocated at random to either the placebo or the treatment group, and the study was performed double blind for 72 hours. A greater 
improvement in forced expiratory volume both before and after treatment with bronchodilators $(p<0.001)$ was seen in the group treated with methylprednisolone.

Several mechanisms might account for the improvement in lung function seen with the corticosteroid. Suppression of the inflammatory response ${ }^{6}$ could have reduced airflow obstruction. ${ }^{7}$ Beta $_{2}$-adrenergic-receptor responsiveness might have increased, ${ }^{8}$ or the steroid-induced euphoria might have resulted in an increased respiratory effort. ${ }^{5}$ Corticosteroids given by mouth also have these properties, and so may be useful additional treatment for patients with chronic bronchitis and intractable symptoms of cough and dyspnoea which persist despite aggressive physiotherapy and bronchodilators.

The rational approach to treating chronic bronchitis is to attempt to achieve maximum reversibility of airways obstruction with bronchodilators and physiotherapy. If this fails to produce stabilisation or improvement a "trial of steroids" may be undertaken. Many physicians are reluctant to prescribe steroids for chronic bronchitis because of the complications associated with this treatment. Since, however, complications relate more to dosage than duration of treatment a suitable regimen for a trial of steroids is oral prednisolone 25-30 mg daily for two to four weeks. ${ }^{4}$ This allows time for a response to develop and should not produce undesirable side effects. Regular objective measurements in the form of spirometry with and without bronchodilators and estimation of arterial gas tensions should be made throughout the trial. If improvement is shown to occur on objective tests, the dosage should be tapered to the lowest level that maintains that improvement. Lack of objective response is a clear indication that treatment should be stopped. Though the result of the work by Albert et $a l^{5}$ is encouraging, the decision to undertake a trial of steroids should be made not indiscriminately but on an individual basis.

${ }^{1}$ Hume KM, Jones ER. Bronchodilators and corticosteroids in asthma. Forced expiratory volume as an aid to diagnosis and treatment. Lancet 1960 ;ii:1319-22.

${ }^{2}$ Clifton $M$, Stuart-Harris $\mathrm{CH}$. Steroid therapy in chronic bronchitis. Lancet $1962 ; \mathrm{i}: 1311-3$.

${ }^{3}$ Freedman BJ. Bronchodilators and corticosteroids in chronic bronchitis and emphysema. $\mathrm{Br} \mathrm{Med} \mathcal{F}$ 1963;ii:1509-12.

4 Sahn SA. Corticosteroids in chronic bronchitis and pulmonary emphysema. Chest 1978;73:389-96.

${ }^{5}$ Albert RK, Martin TR, Lewis SW. Controlled clinical trial of methylprednisolone in patients with chronic bronchitis and acute respiratory insufficiency. Ann Intern Med 1980;92:753-8.

${ }^{6}$ Claman HN. How corticosteroids work. $\mathcal{f}$ Allergy Clin Immunol 1975; 55:145-51.

${ }^{7}$ Cosio M, Ghezzo H, Hogg JC, et al. The relations between structural changes in small airways and pulmonary-function tests. $N$ Engl $\mathcal{F}$ Med 1978;298:1277-81

${ }^{8}$ Townley RG, Reeb R, Fitzgibbons T, Adolphson RL. The effect of corticosteroids on the beta-adrenergic receptors in bronchial smooth muscle. Fournal of Allergy 1970;45:118.

\section{Radiosensitisers}

The radiosensitivity of mammalian celis can be modified by changes in intracellular oxygen tension in vivo and in vitro. Cells that are severely hypoxic are up to three times less susceptible to radiation damage than those that are well oxygenated. Many human tumours have features such as areas of necrosis, large intercapillary distances, and arteriovenous abnormalities, all of which will contribute to a group of cells that are hypoxic yet have the potentiality of growth. Attempts have been made to estimate the effect of this com- ponent, but its importance to the radioresponsiveness of any human tumour is still unknown. In clinical radiotherapy practice fractionation of dosage was used empirically long before studies of the effects of hypoxia in plant and mammalian cells; this fractionation probably permits some reoxygenation of initially hypoxic cells, with cell death and loss initially occurring from the oxygenated compartment.

Given the poor response of many tumours to radiation, despite refinement of techniques using conventional sources of radiotherapy, several approaches have been used to try to enhance the radiosensitivity of tumours while not modifying the amount of damage caused to normal tissues. Three main tactics have been considered: hyperbaric oxygen to increase the oxygen tension in tumour cells during irradiation; use of particles of high linear transfer energy such as fast neutrons; and radiosensitisers.

The results of using hyperbaric oxygen have proved disappointing. Even with unusual fractionation schedules, irradiation of patients within a hyperbaric chamber at three atmospheres pressure has not been associated with a major increase in tumour control rates. The sources of neutrons are few and expensive: clinical trials of the effects of fast neutrons derived from a cyclotron continue in the MRC units at the Hammersmith Hospital and at the Western Infirmary at Edinburgh. In animals hypoxia has been shown to give less protection from the damage caused by neutrons than from conventional photon beams, but in man the benefits conferred by neutron irradiation have not yet been assessed adequately.

Attempts to find a potent and inexpensive chemical sensitiser have led to many different compounds undergoing limited clinical trial, including vitamin $\mathrm{K}$, chemotherapeutic agents such as razoxane (ICRF 159), and, more recently, the nitroimidazoles. These last show high electron affinity, a feature of selective hypoxic cell sensitisers. They can substitute for oxygen: thus they diminish the radioresistance conferred by hypoxia, and do not enhance the radiosensitivity of cells with a normal oxygen tension.

Metronidazole was studied very briefly, but has given way to misonidazole (Ro-07-0582), a compound with greater clinical potential. This drug is stable, not metabolised by cells, and can diffuse into areas of hypoxia. It needs to be present during irradiation to sensitise the cells, which seems to be dependent on the intracellular concentration achieved. ${ }^{1}$ The clinical limits are set by damage to the nervous system, which is dose-related. Like metronidazole, in large doses misonidazole induces nausea and vomiting ${ }^{2}$; repeated high doses may cause convulsions, while lower doses may cause peripheral neuropathy. Though treatment with hepatic enzyme inducers ${ }^{3}$ such as phenytoin and phenobarbitone may reduce the half life of misonidazole, neurotoxicity limits the dose that can be used.

Misonidazole has been used in limited clinical trials in treating adult gliomas, some head and neck tumours, and a few cases of carcinoma of the uterine cervix. These preliminary studies suggest that this particular compound is unlikely to have great clinical efficacy, though much wider evaluation will be needed. Newer drugs in this group, with less neurotoxicity but comparable ability to sensitise hypoxic cells, are being studied in animals, and a more useful sensitiser seems likely to emerge.

While clinical trials may have modified earlier, highly optimistic expectations for the radiosensitisers, interest remains, for they combine ready availability with low price and only partially explored potential. Their weak cytostatic 